\title{
Propiedades Psicométricas del Inventario SISCO-II de Estrés Académico
}

\author{
Psychometric Properties of SISCO-II Inventory of Academic Stress
}

\author{
Juan Luis Castillo-Navarrete ${ }^{1}$, Alejandra Guzmán-Castillo ${ }^{2}$, Claudio Bustos N. ${ }^{3}$, Walter Zavala S. ${ }^{4}$ y \\ Benjamín Vicente P. ${ }^{5}$
}

\section{Resumen}

Estrés académico, proceso multifactorial complejo que alcanza sus grados más elevados a nivel universitario y que para realizar intervenciones efectivas, necesita de instrumentos válidos y fiables para medirlo. El objetivo de este trabajo fue evaluar las propiedades psicométricas de una versión adaptada del inventario SISCO del estrés académico, denominada SISCO-II, destinada a medir estrés académico en estudiantes universitarios. Se aplicó el instrumento a 1.126 estudiantes universitarios de tres universidades chilenas. El análisis factorial confirmatorio mostró buen ajuste a la estructura propuesta de cuatro factores (Estresores, Reacciones físicas y psicológicas, Reacciones del comportamiento social y Afrontamiento). La medida de estrés académico queda constituida por estresores, reacción total (Reacciones físicas, psicológicas y del comportamiento social) y estrategias de afrontamiento. Los resultados avalan la utilidad de SISCO-II para uso diagnóstico y evaluación de intervenciones, siendo una escala válida y confiable.

Palabras clave: estrés académico, Inventario SISCO-II, estresores, somatización, afrontamiento

\begin{abstract}
Academic stress, a complex multifactorial process that reaches its highest levels at the university level and that to carry out effective interventions, needs valid and reliable instruments to measure it. The aim of this work was to evaluate the psychometric properties of an adapted version of the SISCO inventory of academic stress, called SISCO-II, aimed at measuring academic stress in university students. The instrument was applied to 1,126 university students from three Chilean universities. The confirmatory factor analysis showed good adjustment to the proposed structure of four factors (Stressors, Physical and psychological reactions, Social behavior reactions and Coping). The academic stress measure is made up of stressors, total reaction (physical, psychological and social behavior reactions) and coping strategies. The results support the usefulness of SISCO-II for diagnostic use and evaluation of interventions, being a valid and reliable scale.
\end{abstract}

Keywords: academic stress, SISCO-II Inventory, stressors, somatization, coping

\footnotetext{
${ }^{1}$ Candidato a Doctor en Salud Mental, Profesor Asociado, Dpto. de Tecnología Médica, Facultad de Medicina, Universidad de Concepción, Barrio Universitario s/n, Concepción, Región del Bío Bío, Chile. Tel.: 56412661035. Correo: jucastillo@udec.cl

${ }^{2}$ Magíster en Bioquímica clínica e Inmunología, alumna Doctorado en Salud Mental, Universidad de Concepción. Profesor Auxiliar, Departamento de Ciencias Básicas e Histología, Facultad de Medicina, Universidad Católica de la Santísima Concepción. Av. Alonso de Ribera 2850 Concepción, Región del Bío Bío, Chile. Tel.: 56412345592. Correo: aleguzman@ucsc.cl

${ }^{3}$ Doctor en Psicología, Profesor Colaborador, Departamento de Psiquiatría y Salud Mental, Facultad de Medicina, Universidad de Concepción, Av. Juan Bosco s/n 3er piso, Concepción, Región del Bío Bío, Chile. Tel.: 56412312799. Correo: clbustos@udec.cl

${ }^{4}$ Magister en Audiología, Profesor Asociado, Carrera de Fonoaudiología, Facultad de Ciencias de la Salud, Universidad del Desarrollo, Ainavillo 456, Concepción, Región del Bío Bío, Chile. Tel.: +56956176387. Correo: walterzavalasalgado@gmail.com

${ }^{5}$ Doctor en Psiquiatría, Profesor Titular, Departamento de Psiquiatría y Salud Mental, Facultad de Medicina, Universidad de Concepción, Av. Juan Bosco s/n 3er piso, Concepción, Región del Bío Bío, Chile. Tel.: 56412312799. Correo: bvicent@udec.cl

Revista Iberoamericana de Diagnóstico y Evaluación - e Avaliação Psicológica. RIDEP · No56 · Vol.3 · 101-116 · 2020

ISSN: 1135-3848 print /2183-6051online
} 


\section{Introducción}

Todo organismo ante la presencia de un estímulo ambiental produce una respuesta, la cual puede ser definida como estrés (Selye, 1973; Tafet, 2008). El “estrés" es una respuesta neuroendocrina, inmunológica y conductual del organismo a cualquier demanda que se le imponga, y que, a su vez, surge como producto de una interpretación de amenaza o peligro, por lo que permite la adaptación y sobrevivencia de un ser vivo (Selye, 1973). El factor que gatilla esta respuesta adaptativa recibe el nombre de estresor, y según su duración, dará origen a una respuesta de estrés agudo o crónico (Selye, 1973; Tafet, 2008). El estrés es un proceso multifactorial complejo en su génesis fisiopatológica, cuya expresión somática puede incluir trastornos gastrointestinales, insomnio y alteraciones de la memoria, resistencia a la insulina, obesidad, trastornos adaptativos y depresión, entre otras (Tafet \& Nemeroff, 2016). Cuando esto se origina en el contexto de un proceso educativo, se habla de estrés académico (Putwain, 2007).

Se ha demostrado que el estrés académico (EA) ocurre ya en estudiantes de nivel primario, aumentado a medida que se progresa en el nivel de estudios (Putwain, 2007), llegando a sus niveles más elevados en la universidad (Dyson \& Renk, 2006). Por las altas cargas de trabajo, el punto culminante del EA lo representan los estudios superiores. Sin embargo, muchas veces esto también coincide con el proceso de separación de la familia, la incorporación al mercado laboral y la adaptación a un medio poco habitual (Barraza-Macías, 2007a; Putwain, 2007). La población estudiantil universitaria se encuentra sometida a periodos particularmente estresantes, que demandan grandes esfuerzos adaptativos por parte de los estudiantes, los cuales experimentan agotamiento, poco interés ante el estudio, poca autocrítica e incluso dificultades para enfrentar los desafíos propios del entorno.

El uso del término EA suele hacerse con relativa liviandad, aunque sus verdaderos alcances y limitaciones sean desconocidos, situación que se complejiza al enfrentarse a una multiplicidad de términos (por ejemplo estrés estudiantil, estrés universitario, burnout, estrés escolar y estrés de examen) sin una conceptualización clara, con enfoques centrándose en estresores y síntomas (bidimensional), incluso entrecruzándose con otros constructos multidimensionales (ansiedad, vulnerabilidad psicológica, etc.) y además con multiplicidad de instrumentos de evaluación (Barraza-Macías, 2007; 2007b).

El EA ha sido descrito cómo un proceso sistémico de carácter adaptativo y esencialmente psicológico que se presenta descriptivamente en tres etapas: (i) El alumno es sometido a una serie de demandas que, bajo su valoración, son consideradas estresores. (ii) Estos estresores provocan un desequilibrio sistémico (situación estresante) que se manifiesta en una serie de síntomas (indicadores del desequilibrio). (iii) A causa de esto, el alumno a fin de restaurar el equilibrio sistémico realiza una serie de acciones de afrontamiento. En consecuencia, se reconocen tres componentes sistémico-procesuales del EA: (i) Estímulos estresores (input), (ii) síntomas indicadores del desequilibrio sistémico y (iii) Estrategias de afrontamiento (output) (BarrazaMacías, 2006; 2007b; 2007a).

En base a este modelo teórico, se desarrolló un instrumento psicométrico auto descriptivo, el inventario SISCO del estrés académico (SISCOEA), el cual fue estudiado psicométricamente con buenos resultados en México, en base a una estructura de 3 factores con confiabilidades en base a alfa de Cronbach de $0.85,0.91,0.69$ y 0.90 para las dimensiones de estresores, sintomatología, afrontamiento e instrumento completo, respectivamente (Barraza-Macías, 2007b). En Colombia, Malo y colaboradores describen una buena confiabilidad para el instrumento completo (alfa de Cronbach de 0.87) y en base a la estructura de tres factores describen confiabilidad por mitades de $0.76,0.87$ y 0.70 para la dimensión de estresores, sintomatología y afrontamiento, respectivamente (Malo, Cáceres, \& Peña, 2010). En Perú, Manrique y colaboradores reportan confiablidad en base a 3 factores con Omega (Rho de Jöreskog) de $0.82,0.88$ y 0.69 para las dimensiones de estresores, sintomatología y afrontamiento, respectivamente. Además, reportan un análisis confirmatorio en base a 3 y 5 factores con indicadores de ajuste adecuados para solución tanto en base a 3 factores (CFI $=0.95$, RMSA $=0.050$ ) como a 5 factores ( $\mathrm{CFI}=0.96$, RMSA $=0.047$ ) (Manrique-Millones, MillonesRivalles, \& Manrique-Pino, 2019). 
Tabla 1. Distribución de participantes por edad y por sexo, universidad y semestre cursado

\begin{tabular}{lcccccc}
\hline & \multicolumn{2}{c}{ Hombres } & \multicolumn{2}{c}{ Mujeres } & \multicolumn{2}{c}{ Total } \\
\hline Variable & $\mathrm{n}$ & $\%$ & $\mathrm{n}$ & $\%$ & $\mathrm{n}$ & \multicolumn{1}{c}{$\%$} \\
\hline $\mathrm{N}^{\circ}$ Participantes & 287 & 25.49 & 839 & 74.51 & 1126 & 100 \\
UDEC & 153 & 13.59 & 441 & 39.17 & 594 & 52.75 \\
UCSC & 29 & 2.58 & 103 & 9.15 & 132 & 11.72 \\
UDD & 105 & 9.33 & 295 & 26.2 & 400 & 35.52 \\
III-IV semestre & 146 & 12.97 & 393 & 34.9 & 539 & 47.87 \\
V-VI semestre & 130 & 11.55 & 417 & 37.02 & 547 & 48.57 \\
VII-X semestre & 11 & 0.98 & 24 & 2.18 & 35 & 3.11 \\
No declara semestre & 0 & 0 & 5 & 0.44 & 5 & 0.44 \\
\hline Edades participantes & Media & Rango & Media & Rango & Media & Rango \\
\hline UDEC & 21.69 & $19.03-34.85$ & 21.15 & $18.98-50.40$ & 21.29 & $18.98-50.40$ \\
UCSC & 20.92 & $18.97-26.70$ & 20.98 & $18.98-29.03$ & 20.97 & $18.97-29.03$ \\
UDD & 21.72 & $19.52-28.43$ & 21.48 & $18.84-33.30$ & 21.54 & $18.84-33.30$ \\
Total & 21.62 & $19.97-34.85$ & 21.24 & $18.84-50.40$ & 21.34 & $18.84-50.40$ \\
\hline UDEC: Universidad de Concepción. UCSC: Universidad Católica de la Santísima Concepción. UDD: Universidad del & & & & & &
\end{tabular}

SISCO-EA se ha usado en diversas instancias (Correo-Prieto, 2015; Espinel, Robles, \& Álvarez, 2015; Guzmán-Castillo, Sáez, Pérez, \& CastilloNavarrete, 2018; de La Rosa-Rojas et al., 2015; Macías \& Gandara, 2017; Mejía-Rubalcava et al., 2012; Oliván, Boira, \& López del Hoyo, 2011), mostrando buena confiabilidad (alfas de Cronbach entre 0.86 y 0.9 ), a pesar de lo cual son pocos los reportes de análisis de propiedades psicométricas, especialmente en Latinoamérica. Lo anterior es importante, ya que la validación de una escala, inventario o cuestionario implica analizar sus propiedades psicométricas (Martínez, Hernández, \& Hernández, 2006; Nunnally \& Bernstein, 1994). Un instrumento debe someterse, tanto a un análisis de fiabilidad que evalúa el grado de consistencia interna en que un instrumento mide lo que debe medir como también a un análisis de validez, que explora en qué grado un instrumento mide el fenómeno para el cual fue diseñado (Buja \& Eyuboglu, 1992; Hair et al., 2010; Nunnally \& Bernstein, 1994; Tabachnick \& Fidell, 2006). En nuestro país aún no se han estudiado completamente las propiedades psicométricas del inventario SISCO del estrés académico. Al respecto, en la Universidad de Concepción, nuestro grupo ha estudiado algunas propiedades psicométricas del inventario SISCO-EA en estudiantes de la Facultad de Medicina, encontrando buenos indicadores de fiabilidad (alfa de Cronbach de 0.86) faltando aún confirmar el análisis psicométrico realizado (Guzmán-Castillo, Sáez, Pérez, \& Castillo-Navarrete, 2018).

El objetivo del presente artículo es estudiar las propiedades psicométricas y adaptar el inventario
SISCO para estrés académico, en una nueva versión, SISCO-II, en estudiantes universitarios chilenos.

\section{Método}

\section{Muestra}

La muestra estuvo conformada por 1.126 estudiantes de segundo y tercer año pertenecientes a la Universidad de Concepción (UdeC), Universidad Católica de la Santísima Concepción (UCSC) y Universidad del Desarrollo campus Concepción (UDD), Chile. Los estudiantes que respondieron el instrumento firmaron un formulario de consentimiento informado y completaron una ficha de datos generales.

\section{Descripción de la muestra}

La distribución de mujeres y hombres fue de $74.51 \%$ (839) y $25.49 \%$ (287) respectivamente. La proporción de participantes de acuerdo con la universidad de la cual provenían fue de $52.75 \%$ (594) para la UdeC, $11.72 \%$ (132) para la UCSC y $35.52 \%$ (400) para la UDD. La media de edad fue de 21.34 años (18.84-50.4) para la muestra total y una media de edad por sexo de 21.24 años (18.8450.4) para mujeres y 21.62 años (18.97-34.85) para hombres (Tabla 1).

\section{Instrumento}

El inventario SISCO-EA original (BarrazaMacías, 2007b; Jaimes, 2008), en idioma español, contiene 31 ítems, siendo el primero de ellos, una parte inicial de filtro, en términos dicotómicos (si - no) que permiten determinar si el encuestado 
Tabla 2. Estadísticos descriptivos de todos los ítems de la versión modificada del Inventario SISCO del estrés académico

\begin{tabular}{|c|c|c|c|c|c|c|c|c|c|}
\hline \multirow{2}{*}{\multicolumn{2}{|c|}{ Ítems }} & \multicolumn{4}{|c|}{ Análisis Factorial Exploratorio } & \multicolumn{4}{|c|}{ Análisis Factorial Confirmatorio } \\
\hline & & Media & DS & Simetría & Kurtosis & Media & DS & Simetría & Kurtosis \\
\hline 3.1 & La competencia con los compañeros del grupo & 2.48 & 1.04 & 0.41 & -0.29 & 2.46 & 1.05 & 0.37 & -0.38 \\
\hline 3.2 & Sobrecarga de tareas y trabajos académicos & 3.86 & 0.83 & -0.21 & -0.39 & 3.88 & 0.85 & -0.45 & -0.004 \\
\hline 3.3 & La personalidad y carácter de los profesores & 2.75 & 0.94 & 0.29 & 0.008 & 2.78 & 0.99 & 0.30 & -0.15 \\
\hline 3.4 & $\begin{array}{l}\text { Las evaluaciones de los profesores (exámenes, ensayos, } \\
\text { trabajos de investigación, etc.) }\end{array}$ & 3.86 & 0.84 & -0.32 & -0.29 & 3.93 & 0.89 & -0.55 & 0.00 \\
\hline 3.5 & $\begin{array}{l}\text { El tipo de trabajo que te piden los profesores (consulta de } \\
\text { mapas, fichas de trabajo, ensayos, mapas conceptuales, } \\
\text { etc.) }\end{array}$ & 3.25 & 0.97 & 0.03 & -0.40 & 3.24 & 1.00 & 0.03 & -0.46 \\
\hline 3.6 & No entender los temas que se abordan en la clase & 3.07 & 1.06 & 0.21 & -0.50 & 3.05 & 1.04 & 0.18 & -0.37 \\
\hline 3.7 & $\begin{array}{l}\text { Participación en clase (responder a preguntas, } \\
\text { exposiciones, etc.) }\end{array}$ & 2.76 & 1.09 & 0.29 & -0.51 & 2.76 & 1.11 & 0.27 & -0.48 \\
\hline 3.8 & Tiempo limitado para hacer el trabajo & 3.54 & 1.00 & -0.29 & -0.41 & 3.56 & 1.03 & -0.19 & -0.69 \\
\hline 3.9 & $\begin{array}{l}\text { Los compañeros de grupo progresan más rápido en tareas } \\
\text { y/o trabajos académicos }(*) \text {. }\end{array}$ & 2.66 & 1.04 & 0.32 & -0.32 & 2.59 & 1.15 & 0.47 & -0.41 \\
\hline 4.1 & Trastornos del sueño (insomnio o pesadillas) & 3.00 & 1.09 & -0.07 & -0.48 & 2.95 & 1.14 & -0.04 & -0.69 \\
\hline 4.2 & Fatiga crónica (cansancio permanente) & 3.40 & 1.09 & -0.30 & -0.51 & 3.43 & 1.14 & -0.39 & -0.58 \\
\hline 4.3 & Dolores de cabeza o migrañas & 3.02 & 1.12 & 0.02 & -0.72 & 3.04 & 1.14 & -0.08 & -0.65 \\
\hline 4.4 & Problemas de digestión, dolor abdominal o diarrea & 2.71 & 1.17 & 0.24 & -0.77 & 2.84 & 1.26 & 0.13 & -1.00 \\
\hline 4.5 & Rascarse, morderse las uñas, frotarse, etc. & 3.03 & 1.41 & -0.06 & -1.30 & 3.09 & 1.44 & -0.12 & -1.31 \\
\hline 4.6 & Somnolencia o mayor necesidad de dormir. & 3.77 & 1.09 & -0.61 & -0.40 & 3.76 & 1.10 & -0.61 & -0.39 \\
\hline 4.7 & Dolores musculares y/o contracturas & 2.97 & 1.23 & 0.002 & -0.92 & 3.00 & 1.25 & -0.05 & -0.99 \\
\hline 4.8 & Reacciones cutáneas (sarpullido, descamación, etc.) & 1.99 & 1.21 & 1.02 & -0.08 & 2.11 & 1.27 & 0.90 & -0.32 \\
\hline 4.9 & Inquietud (incapacidad de relajarse y estar tranquilo) & 3.48 & 1.00 & -0.27 & -0.45 & 3.41 & 1.07 & -0.17 & -0.65 \\
\hline 4.10 & Sentimientos de depresión y tristeza (decaído) & 3.03 & 1.15 & -0.05 & -0.79 & 3.01 & 1.19 & 0.06 & -0.85 \\
\hline 4.11 & Ansiedad, angustia o desesperación & 3.34 & 1.09 & -0.16 & -0.68 & 3.32 & 1.14 & -0.17 & -0.77 \\
\hline 4.12 & Sentimiento de agresividad o aumento de irritabilidad & 2.70 & 1.14 & 0.22 & -0.72 & 2.74 & 1.25 & 0.24 & -0.94 \\
\hline 4.13 & Cambios bruscos de humor & 2.77 & 1.13 & 0.22 & -0.70 & 2.83 & 1.26 & 0.14 & -0.99 \\
\hline 4.14 & Conflictos o tendencia a polemizar o discutir & 2.27 & 1.02 & 0.62 & 0.006 & 2.30 & 1.12 & 0.63 & -0.37 \\
\hline 4.15 & Aislamiento de los demás & 2.42 & 1.14 & 0.44 & -0.58 & 2.46 & 1.18 & 0.44 & -0.71 \\
\hline 4.16 & Desgano para realizar tus labores de estudiante & 3.18 & 1.00 & 0.007 & -0.33 & 3.21 & 1.05 & -0.11 & -0.43 \\
\hline 4.17 & Aumento o reducción del consumo de alimentos & 3.36 & 1.19 & -0.33 & -0.77 & 3.37 & 1.22 & -0.35 & -0.82 \\
\hline 5.1 & $\begin{array}{l}\text { Habilidad para defender nuestras preferencias } 0 \\
\text { sentimientos sin dañar a otros) }\end{array}$ & 3.36 & 1.00 & -0.31 & -0.24 & 3.31 & 0.99 & -0.25 & -0.40 \\
\hline 5.2 & Elaboración de un plan de ejecución de sus tareas & 3.30 & 1.00 & -0.11 & -0.51 & 3.30 & 1.00 & -0.19 & -0.41 \\
\hline 5.3 & Elogios a sí mismo & 2.37 & 1.06 & 0.52 & -0.39 & 2.40 & 1.15 & 0.62 & -0.39 \\
\hline 5.4 & Práctica religiosa (oraciones o asistencia a iglesia/templo) & 2.27 & 1.38 & 0.77 & -0.70 & 2.28 & 1.39 & 0.65 & -0.97 \\
\hline 5.5 & Búsqueda de información sobre la situación & 2.20 & 1.11 & 0.61 & -0.47 & 2.24 & 1.11 & 0.53 & -0.55 \\
\hline 5.6 & $\begin{array}{l}\text { Ventilación y confidencias (verbalización de la situación } \\
\text { que preocupa) }\end{array}$ & 2.89 & 1.05 & 0.12 & -0.54 & 2.84 & 1.15 & 0.08 & -0.80 \\
\hline 5.7 & $\begin{array}{l}\text { Intenté sacar algo positivo o beneficioso de la situación } \\
\text { estresante }\end{array}$ & 3.16 & 1.11 & -.11 & -.71 & 3.12 & 1.16 & -0.11 & -0.76 \\
\hline 5.8 & Consumo de sustancias (Café, energéticas, tabaco, etc.) & 3.13 & 1.40 & -.17 & -1.23 & 3.08 & 1.45 & -0.10 & -1.33 \\
\hline 5.9 & $\begin{array}{l}\text { Practicar un pasatiempo (act. Física, leer, ver series, redes } \\
\text { sociales, etc.) }\end{array}$ & 3.52 & 1.13 & -.32 & -.71 & 3.62 & 1.17 & -0.55 & -0.51 \\
\hline 5.10 & $\begin{array}{l}\text { Acompañarse de un ser querido (familia, mascotas, } \\
\text { amigos, etc.) }\end{array}$ & 3.58 & 1.21 & -.49 & -.72 & 3.53 & 1.21 & -0.47 & -0.70 \\
\hline
\end{tabular}
DS: Desviación estándar

continúa o no respondiendo el inventario. Una segunda parte con una única pregunta que apunta a identificar el nivel de autopercepción global del nivel de estrés académico. La tercera, cuarta y quinta parte corresponde a las dimensiones de estresores (08 ítems), sintomatología (14 ítems) y afrontamiento (06 ítems), respectivamente. Desde la segunda a la quinta parte se emplea una escala tipo Likert de cincos puntos que da cuenta de la frecuencia de cada situación o conducta (1: nunca; 2: rara vez; 3: algunas veces, 4: casi siempre; 5: siempre).

Considerando un estudio anterior dónde se analizó la estructura factorial, así como la confiabilidad de cada una de las dimensiones del inventario SISCO del estrés académico (GuzmánCastillo, Sáez, Pérez, \& Castillo-Navarrete, 2018), se reunió a un grupo de estudiantes universitarios de tercer año a fin de preguntarles si los ítems que componen cada una de las dimensiones, representan la forma en que perciben el estrés académico. De igual forma se les solicitó identificar situaciones estresoras, de somatización $\mathrm{y}$ afrontamiento que no estuvieran consideradas en el instrumento. De esta forma se agregaron: 01 ítems a la dimensión de estresores, 03 ítems a la dimensión de somatización y 04 ítems a la dimensión de afrontamiento. La versión modificada del inventario SISCO-EA quedó compuesta por un total de 38 ítems distribuidos en 02 preguntas iniciales, 09 ítems en la dimensión de estresores, 17 ítems en la dimensión de somatización y 10 en la dimensión de afrontamiento (Tabla 2).

\section{Procedimiento}

La aplicación del inventario en su forma modificada (inventario SISCO del estrés 
académico original más los nuevos ítems) fue conducida por profesionales del equipo investigador al inicio de una clase (previa autorización y coordinación de las autoridades y docentes correspondientes) de segundo y tercer año de las carreras Tecnología Médica, Obstetricia y Puericultura, Kinesiología, Fonoaudiología, Química y Farmacia, Nutrición y Dietética, Piscología, Bachillerato en Humanidades, Traducción e Interpretación y Licenciatura en artes visuales de la UdeC, Tecnología Médica y Enfermería de la UCSC y Fonoaudiología, Kinesiología, Enfermería, Nutrición y Dietética y Odontología de la UDD. La aplicación del instrumento tomó un tiempo de 10 minutos aproximadamente. Los participantes que respondieron el instrumento fueron voluntarios y daban fe de ello firmando un consentimiento informado, el cual fue revisado y aprobado por el Comité Ético Científico de la Facultad de Medicina de la UdeC. La aplicación del instrumento se realizó durante el periodo comprendido entre junio y noviembre de 2017.

\section{Análisis Estadísticos}

Para evaluar la estructura factorial, se separó la muestra total en dos partes, la primera submuestra $(n=563)$ se utilizó para el análisis factorial exploratorio (AFE), cuya solución fue probada mediante un análisis factorial confirmatorio (AFC) sobre la segunda submuestra $(n=563)$ (Ledesma, Ferrando \& Tosi, 2019).

Para analizar la adecuación del AFE a los datos recolectados, se empleó la prueba de esfericidad de Bartlett y la prueba de adecuación muestral de Kaiser Meyer Olkin (KMO) (Kaiser, 1974; Ledesma, Ferrando, \& Tosi, 2019). Para determinar el número de factores, se empleó el Análisis Paralelo de Horn, basado en un remuestreo no paramétrico de 10.000 muestras bootstrapping (Horn, 1965; Ledesma, Ferrando, \& Tosi 2019). Una vez determinado el número de factores, se generó una solución utilizando como método de extracción de factores, el método de mínimos cuadrados con rotación Oblimin.

A partir de la solución factorial inicial, se buscó obtener una estructura simple, dónde cada ítem tuviese una carga factorial importante sólo en un factor. Se consideró como carga factorial mínima un valor de 0.3 (Bruna et al., 2017; Fasce et al. 2011; Guzmán-Castillo, Sáez, Pérez, \& Castillo-Navarrete, 2018; Hair et al., 2010; Martínez et al., 2006; Pérez et al., 2015). Si las cargas factoriales de un ítem eran menores a 0.3 en todos los factores, o superior a 0.3 en dos o más factores, se procedió a evaluar la pertinencia conceptual de eliminarlo, y de ser así, proceder a una nueva solución factorial (Guzmán-Castillo, Sáez, Pérez, \& Castillo-Navarrete, 2018). Ya identificados los factores, se calculó la confiabilidad, mediante alfa de Cronbach y omega, para cada uno de ellos a fin de estimar su consistencia interna.

Una vez realizado el análisis factorial exploratorio con la primera submuestra, con la otra submuestra se realizó un análisis factorial confirmatorio con estimación WLSMV, apta para ítems a nivel ordinal considerando como adecuados valores de CFI y TLI mayores o iguales a 0.95 y un RMSEA menor a 0.06 con un p>.05 (Bruna et al., 2017; Cova et al., 2019). Finalmente, se evaluó la correlación entre los factores aplicando el coeficiente de Pearson. En todos los análisis se consideró un nivel de significación de $\alpha=0.05$. Para el análisis de los datos, estos se codificaron en Microsoft Excel 2016 y su procesamiento se realizó con el paquete estadístico R (Versión 3).

\section{Resultados}

En la Tabla 2 se muestran los estadísticos descriptivos para todos los ítems de la versión modificada del inventario SISCO-EA a fin de establecer si existe alguno con asimetría o curtosis muy grande que deba ser eliminado. El ítem 4.5 presentó una curtosis menor a -1 y los ítems 4.8 y 5.8 presentaron una asimetría mayor a 1 y una curtosis menor a -1, respectivamente. Considerando las desviaciones estándar de estos ítems y que teóricamente son importantes, no son eliminados y se procede a trabajar con la matriz de correlaciones de Pearson.

Para el análisis factorial exploratorio, primero se procedió a evaluar la pertinencia de este, analizando la matriz de correlaciones policóricas sobre la mitad de la muestra seleccionada para este proceso. El estadístico de adecuación muestral de Kaiser-Myer-Olkin (KMO) fue de 
Tabla 3. Matriz de cargas factoriales por ítem obtenida en base a solución inicial de 3 y 4 factores (36 ítems)

\begin{tabular}{|c|c|c|c|c|c|c|c|c|}
\hline \multirow{2}{*}{\multicolumn{2}{|c|}{ Ítems }} & \multicolumn{3}{|c|}{3 Factores } & \multicolumn{4}{|c|}{4 Factores } \\
\hline & & F1 & F2 & F3 & F1 & F2 & F3 & $\mathrm{F} 4$ \\
\hline$\overline{3.1}$ & La competencia con los compañeros del grupo $^{\dagger}$ & 0.17 & 0.22 & -0.06 & 0.18 & 0.17 & 0.05 & -0.04 \\
\hline 3.3 & La personalidad y carácter de los profesores & 0.16 & 0.38 & 0.04 & 0.05 & 0.44 & -0.02 & 0.01 \\
\hline 3.4 & $\begin{array}{l}\text { Las evaluaciones de los profesores (exámenes, ensayos, } \\
\text { trabajos de investigación, etc.) }\end{array}$ & 0.08 & 0.61 & 0.05 & 0.02 & 0.67 & -0.01 & -0.01 \\
\hline 3.5 & $\begin{array}{l}\text { El tipo de trabajo que te piden los profesores (consulta de } \\
\text { mapas, fichas de trabajo, ensayos, mapas conceptuales, } \\
\text { etc.) }\end{array}$ & -0.01 & 0.64 & 0.03 & -0.04 & 0.73 & 0.00 & 0.03 \\
\hline 3.6 & No entender los temas que se abordan en la clase & -0.10 & 0.61 & -0.17 & 0.05 & 0.51 & -0.02 & -0.13 \\
\hline 3.7 & $\begin{array}{l}\text { Participación en clase (responder a preguntas, } \\
\text { exposiciones, etc.) }\end{array}$ & 0.04 & 0.42 & -0.15 & 0.11 & 0.30 & -0.01 & -0.16 \\
\hline 3.8 & Tiempo limitado para hacer el trabajo & 0.10 & 0.56 & 0.05 & -0.03 & 0.66 & 0.03 & -0.01 \\
\hline 3.9 & $\begin{array}{l}\text { Los compañeros de grupo progresan más rápido en tareas } \\
\text { y/o trabajos académicos. }\left(^{*}\right)\end{array}$ & 0.17 & 0.36 & -0.12 & 0.15 & 0.38 & 0.02 & -0.11 \\
\hline \multicolumn{9}{|c|}{ Sintomatología } \\
\hline 4.5 & Rascarse, morderse las uñas, frotarse, etc. & 0.40 & 0.01 & 0.08 & 0.35 & -0.02 & 0.05 & -0.01 \\
\hline 4.6 & Somnolencia o mayor necesidad de dormir. & 0.52 & 0.09 & 0.16 & 0.43 & 0.18 & 0.04 & 0.00 \\
\hline 4.7 & Dolores musculares y/o contracturas $(*)$ & 0.47 & 0.06 & 0.12 & 0.51 & 0.05 & 0.00 & 0.09 \\
\hline 4.8 & Reacciones cutáneas (sarpullido, descamación, etc.) $(*)$ & 0.41 & -0.05 & 0.06 & 0.42 & -0.08 & 0.07 & -0.02 \\
\hline 4.9 & Inquietud (incapacidad de relajarse y estar tranquilo) & 0.53 & 0.20 & 0.01 & 0.43 & 0.13 & 0.22 & 0.02 \\
\hline 4.10 & Sentimientos de depresión y tristeza (decaído) & 0.66 & 0.06 & -0.09 & 0.47 & 0.03 & 0.40 & -0.12 \\
\hline 4.11 & Ansiedad, angustia o desesperación & 0.63 & 0.11 & 0.06 & 0.46 & 0.12 & 0.28 & 0.02 \\
\hline 4.12 & Sentimiento de agresividad o aumento de irritabilidad & 0.69 & -0.03 & -0.03 & 0.00 & 0.06 & 0.76 & 0.08 \\
\hline 4.13 & Cambios bruscos de humor $(*)$ & 0.74 & -0.09 & -0.05 & 0.15 & 0.00 & 0.66 & -0.01 \\
\hline 4.14 & Conflictos o tendencia por polemizar o discutir & 0.66 & -0.14 & -0.09 & -0.05 & 0.04 & 0.68 & 0.05 \\
\hline 4.15 & Aislamiento de los demás & 0.54 & -0.05 & -0.27 & 0.06 & -0.09 & 0.59 & -0.15 \\
\hline 4.16 & Desgano para realizar tus labores de estudiante & 0.54 & 0.06 & -0.11 & 0.13 & 0.11 & 0.46 & -0.07 \\
\hline$\underline{4.17}$ & Aumento o reducción del consumo de alimentos & 0.50 & 0.05 & 0.11 & 0.39 & 0.08 & 0.15 & 0.03 \\
\hline 5.6 & $\begin{array}{l}\text { Ventilación y confidencias (verbalización de la situación } \\
\text { que preocupa) }\end{array}$ & 0.03 & 0.05 & 0.48 & 0.02 & 0.12 & 0.12 & 0.51 \\
\hline 5.7 & $\begin{array}{l}\text { Intenté sacar algo positivo o beneficioso de la situación } \\
\text { estresante }(*)\end{array}$ & -0.01 & -0.14 & 0.55 & 0.10 & -0.13 & -0.04 & 0.57 \\
\hline 5.8 & $\begin{array}{l}\text { Consumo de sustancias (Café, energéticas, tabaco, etc.) }(*) \\
+\end{array}$ & 0.25 & 0.11 & 0.19 & 0.14 & 0.19 & 0.04 & 0.11 \\
\hline 5.9 & $\begin{array}{l}\text { Practicar un pasatiempo (act. Física, leer, ver series, redes } \\
\text { sociales, etc.) }(*)\end{array}$ & 0.03 & -0.18 & 0.23 & -0.21 & -0.10 & 0.15 & 0.32 \\
\hline 5.10 & $\begin{array}{l}\text { Acompañarse de un ser querido (familia, mascotas, } \\
\text { amigos, etc.) }(*)\end{array}$ & -0.01 & -0.05 & 0.38 & -0.05 & 0.08 & -0.06 & 0.40 \\
\hline
\end{tabular}

$(\dagger)$ Ítems eliminados en la solución final de 4 factores, $\left(^{*}\right)$ Corresponden a ítems de reciente incorporación; Las cargas factoriales superiores a 0.3 se muestran en negrita y cursiva.

0.91, muy bueno para el análisis factorial. A su vez, la prueba de esfericidad de Bartlett fue significativa $\left(X^{2}(630)=5874, p<.001\right)$, indicando que hay correlaciones entre los ítems en la población. Se comienza probando una solución de tres factores, considerando que el inventario SISCO del estrés académico en su forma original considera tres factores correspondientes a las dimensiones de estresores, somatización o sintomatología y estrategias de afrontamiento, la cual explicó un $28 \%$ de la varianza. Luego se consideraron aquellos ítems con cargas factoriales menores a 0.3 , para proceder a eliminar el ítem con menor carga factorial y repetir la solución factorial. De esta forma se eliminaron los ítems 3.1 (La competencia con los compañeros del grupo) de la dimensión de estresores y los ítems
5.4 (Práctica religiosa (oraciones o asistencia a iglesia/templo)), 5.5 (Búsqueda de información sobre la situación), 5.8 (Consumo de sustancias (Café, energéticas, tabaco, etc.)) y 5.9 (Practicar un pasatiempo (actividad física, leer, ver series, redes sociales, etc.)) de la dimensión de afrontamiento.

Considerando la solución factorial obtenida se definieron 3 factores (Tabla 3), factor 1 correspondiente a sintomatología, factor 2 correspondiente a estresores $y$ factor 3 correspondiente a estrategias de afrontamiento. En cuanto a la correlación de estos factores (Figura 1), estresores se correlaciona con somatización $(\mathrm{r}=0.56)$ no así con afrontamiento $(\mathrm{r}=-0.03)$ y este a su vez tampoco se correlaciona con somatización $(\mathrm{r}=-0.06)$. La dimensión de estresores presentó una buena confiabilidad (alfa 


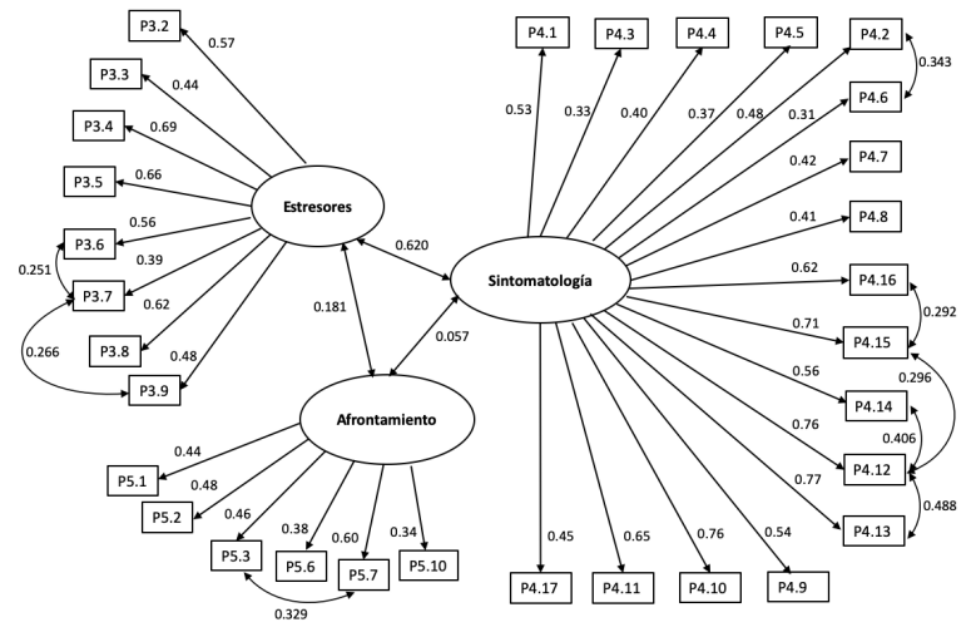

Figura 1. Gráfico de estimadores de los parámetros del modelo en base a solución de 3 factores

Tabla 4. Indicadores de ajuste del análisis Factorial Confirmatorio identificado 3 y 4 factores (submuestra 2,

\begin{tabular}{|c|c|c|c|c|c|c|}
\hline \multicolumn{7}{|c|}{$\mathrm{n}=563)$} \\
\hline Modelo & $\mathrm{X}^{2}$ & $\mathrm{X}^{2} / \mathrm{gl}$ & CFI & TLI & SRMR & RMSEA \\
\hline \multirow{3}{*}{$\begin{array}{l}3 \text { factores } \\
\text { Exploratorio inicial, (36 ítems) } \\
\text { Exploratorio reducido, (31 } \\
\text { ítems) }\end{array}$} & & & & & & \\
\hline & $\mathrm{X}^{2}(423)=1081.07, p=.000$ & 2.556 & 0.934 & 0.927 & 0.076 & $0.054[0.050,0.073], p=.063$ \\
\hline & $\mathrm{X}^{2}(431)=1344.23, p<.000$ & 3.119 & 0.908 & 0.901 & 0.068 & $0.063[0.059,0.066], p<.000$ \\
\hline \multicolumn{7}{|l|}{4 factores } \\
\hline \multirow{2}{*}{$\begin{array}{l}\text { Exploratorio inicial, ( } 37 \text { ítems) } \\
\text { Exploratorio reducido, ( } 31 \\
\text { ítems) }\end{array}$} & $X^{2}(587)=1954.42, p=.000$ & 3.330 & 0.863 & 0.853 & 0.072 & $0.066[0.063,0.070], p=.000$ \\
\hline & $\mathrm{X}^{2}(424)=891.86, p<.000$ & 2.103 & 0.952 & 0.947 & 0.058 & $0.045[0.041,0.049], p=.969$ \\
\hline
\end{tabular}

Nota. Los modelos se encuentran anidados entre sí. El intervalo de confianza de RMSEA es al $10 \%$.

de Cronbach $=0.77$ y Omega $=0.78$ ), la dimensión de somatización a su vez presentó una muy buena confiabilidad (alfa de Cronbach $=0.89$ y Omega $=0.89$ ), mientras que la dimensión de afrontamiento, no presentó una buena confiabilidad (alfa de Cronbach $=0.58$ y Omega $=0.58$ ), lo cual concuerda con lo que reportamos previamente (Guzmán-Castillo, Sáez, Pérez, \& Castillo-Navarrete, 2018).

En la Tabla 4 se pueden ver los indicadores de ajuste del análisis factorial confirmatorio en base a 3 factores, dónde el modelo que proviene del análisis factorial exploratorio inicial obtenido al realizar el análisis a los 36 ítems presentó valores de CFI y TLI menores al 0.95 esperado con un RMSEA significativo $(0.054, p=.000)$. El modelo exploratorio con los 31 ítems finales presentó indicadores de ajuste adecuados, aunque CFI y TLI fueron menores al 0.95 esperado $(0.908 \mathrm{y}$ 0.901 , respectivamente).

Los estimadores para los parámetros del modelo se presentan en la Figura 1 pudiendo apreciarse las correlaciones entre los factores. El factor 1 (somatización) se correlaciona significativamente con el Factor 2 correspondiente a estresores $(r=0.620)$. A su vez, el factor 2 presenta una baja correlación con el factor 3 correspondiente a estrategias de afrontamiento $(\mathrm{r}=0.181)$, el cual no se correlaciona con el factor $1(\mathrm{r}=0.057)$. También es posible apreciar la correlación existente entre los ítems 3.6 con $3.7(\mathrm{r}=0.251)$ y este último con 3.9 $(\mathrm{r}=0.266)$, todos pertenecientes la dimensión de estresores. En la dimensión de somatización es posible ver correlaciones entre los ítems 4.2 con 4.6 $(\mathrm{r}=0.343), 4.16$ con 4.15 ( $\mathrm{r}=0.292), 4.12$ con 4.15 $(\mathrm{r}=0.296), 4.12$ con $4.13(\mathrm{r}=0.488)$ y 4.12 con 4.14 (0.406). En el caso de la dimensión de estrategias de afrontamiento se puede apreciar correlación entre los ítems 5.3 y 5.7 ( $\mathrm{r}=0.329)$.

Producto de lo anterior y considerando que los indicadores de ajuste obtenido no son óptimos, con valores de CFI y TLI menores al 0.95 , pero un con RMSEA aceptable (RMSEA $=0.063, p<.000$ ) se decidió probar la solución factorial con 4 factores obtenida con el análisis paralelo de Horn, solución que explicó un $32 \%$ de la varianza. Considerando los ítems que presentaron cargas factoriales menores a 0.3 para todos los factores, se procedió a eliminar el ítem con menor carga factorial y repetir la solución factorial. Bajo este criterio, se repitió el mismo procedimiento hasta encontrar una solución estable, que explicó 
Tabla 5. Matriz de cargas factoriales por ítem en base a solución de 4 factores (32 ítems)

\begin{tabular}{|c|c|c|c|c|c|c|c|c|c|}
\hline & Factor & & Ítems & F1 & $\mathrm{F} 2$ & F3 & F4 & & \\
\hline & Estresores & 3.2 & Sobrecarga de tareas y trabajos académicos & 0.11 & 0.56 & 0.10 & -0.01 & \multirow{26}{*}{ 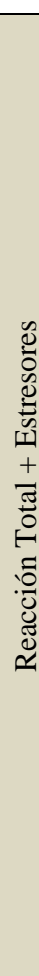 } & \\
\hline & (Factor 2) & 3.3 & La personalidad y carácter de los profesores & 0.06 & 0.43 & 0.03 & -0.03 & & \\
\hline & & 3.4 & $\begin{array}{l}\text { Las evaluaciones de los profesores (exámenes, ensayos, } \\
\text { trabajos de investigación, etc.) }\end{array}$ & 0.01 & 0.68 & 0.00 & 0.00 & & \\
\hline & & 3.5 & $\begin{array}{l}\text { El tipo de trabajo que te piden los profesores (consulta de } \\
\text { mapas, fichas de trabajo, ensayos, mapas conceptuales, etc.) }\end{array}$ & -0.02 & 0.71 & 0.03 & -0.02 & & \\
\hline & & 3.6 & No entender los temas que se abordan en la clase & -0.01 & 0.54 & -0.14 & 0.02 & & \\
\hline & & 3.7 & $\begin{array}{l}\text { Participación en clase (responder a preguntas, exposiciones, } \\
\text { etc.) }\end{array}$ & 0.04 & 0.33 & -0.18 & 0.03 & & \\
\hline & & 3.8 & Tiempo limitado para hacer el trabajo & -0.02 & 0.66 & 0.00 & 0.02 & & \\
\hline & & 3.9 & $\begin{array}{l}\text { Los compañeros de grupo progresan más rápido en tareas y/o } \\
\text { trabajos académicos. }(*)\end{array}$ & 0.05 & 0.40 & -0.14 & 0.09 & & 过 \\
\hline \multirow{24}{*}{ 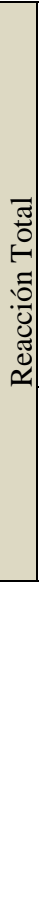 } & Reacciones físicas & 4.1 & Trastornos del sueño (insomnio o pesadillas) & 0.60 & -0.03 & -0.02 & 0.10 & & \\
\hline & y psicológicas & 4.2 & Fatiga crónica (cansancio permanente) & 0.63 & 0.09 & -0.02 & 0.03 & & $\varangle$ \\
\hline & (Factor 1) & 4.3 & Dolores de cabeza o migrañas & 0.64 & 0.03 & -0.02 & -0.13 & & 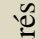 \\
\hline & & 4.4 & Problemas de digestión, dolor abdominal o diarrea & 0.58 & -0.03 & 0.08 & -0.06 & & Eี \\
\hline & & 4.5 & Rascarse, morderse las uñas, frotarse, etc. & 0.34 & -0.02 & -0.02 & 0.06 & & 出 \\
\hline & & 4.6 & Somnolencia o mayor necesidad de dormir. & 0.48 & 0.15 & 0.00 & 0.01 & & $\tau$ \\
\hline & & 4.7 & Dolores musculares y/o contracturas $(*)$ & 0.53 & 0.04 & 0.10 & 0.00 & & $\underset{\pi}{\pi}$ \\
\hline & & 4.8 & Reacciones cutáneas (sarpullido, descamación, etc.) (*) & 0.40 & -0.07 & -0.04 & 0.08 & & ?] \\
\hline & & 4.9 & Inquietud (incapacidad de relajarse y estar tranquilo) & 0.40 & 0.14 & 0.01 & 0.25 & & $\sum$ \\
\hline & & 4.10 & Sentimientos de depresión y tristeza (decaído) $(* *)$ & 0.44 & 0.03 & -0.13 & 0.41 & & \\
\hline & & 4.11 & Ansiedad, angustia o desesperación & 0.44 & 0.13 & 0.02 & 0.29 & & \\
\hline & & 4.17 & Aumento o reducción del consumo de alimentos & 0.39 & 0.08 & 0.02 & 0.15 & & \\
\hline & \multirow{6}{*}{$\begin{array}{l}\text { Reacciones del } \\
\text { comportamiento } \\
\text { social } \\
\text { (Factor } 4)\end{array}$} & 4.10 & Sentimientos de depresión y tristeza (decaído) $(* *)$ & 0.44 & 0.03 & -0.13 & 0.41 & & \\
\hline & & 4.12 & Sentimiento de agresividad o aumento de irritabilidad & -0.01 & 0.05 & 0.08 & 0.77 & & \\
\hline & & 4.13 & Cambios bruscos de humor $(*)$ & 0.15 & -0.01 & -0.01 & 0.66 & & \\
\hline & & 4.14 & Conflictos o tendencia a polemizar o discutir & -0.06 & 0.03 & 0.06 & 0.69 & & \\
\hline & & 4.15 & Aislamiento de los demás & 0.01 & -0.08 & -0.17 & 0.62 & & \\
\hline & & 4.16 & Desgano para realizar tus labores de estudiante & 0.14 & 0.11 & -0.08 & 0.44 & & \\
\hline & \multirow{6}{*}{$\begin{array}{l}\text { Estrategias de } \\
\text { afrontamiento } \\
\text { (Factor 3) }\end{array}$} & 5.2 & Elaboración de un plan de ejecución de sus tareas & 0.15 & 0.10 & 0.38 & -0.09 & & \\
\hline & & 5.3 & Elogios a sí mismo & -0.14 & -0.03 & 0.45 & 0.09 & & \\
\hline & & 5.6 & $\begin{array}{l}\text { Ventilación y confidencias (verbalización de la situación que } \\
\text { preocupa) }\end{array}$ & 0.00 & 0.14 & 0.47 & 0.15 & & \\
\hline & & 5.7 & $\begin{array}{l}\text { Intenté sacar algo positivo o beneficioso de la situación } \\
\text { estresante }(*)\end{array}$ & 0.12 & -0.13 & 0.54 & -0.04 & & \\
\hline & & 5.9 & $\begin{array}{l}\text { Practicar un pasatiempo (act. Física, leer, ver series, redes } \\
\text { sociales, etc.) }(*)\end{array}$ & -0.14 & -0.11 & 0.34 & 0.10 & & \\
\hline & & 5.10 & $\begin{array}{l}\text { Acompañarse de un ser querido (familia, mascotas, amigos, } \\
\text { etc.) }(*)\end{array}$ & -0.01 & 0.08 & 0.42 & -0.08 & & \\
\hline
\end{tabular}

(*) Corresponden a nuevos ítems; (**) Ítem presenta carga cruzada.

Tabla 6. Estadísticos descriptivos y confiabilidad de los factores identificados en base a solución de 4 factores

\begin{tabular}{lccccccc}
\hline & $\mathrm{n}$ & Media & SD & Sesgo & Curtosis & $\alpha$ Cronbach & Omega \\
\hline Estresores & 563 & 3.220 & 0.615 & -0.086 & 0.290 & 0.7815 & 0.7792 \\
Reacciones físicas y psicológicas & 563 & 3.091 & 0.713 & -0.048 & -0.485 & 0.8516 & 0.8515 \\
Reacciones del comportamiento social & 563 & 2.728 & 0.815 & 0.289 & -0.309 & 0.8372 & 0.8402 \\
Estrategias de afrontamiento & 563 & 3.137 & 0.623 & 0.0121 & -0.281 & 0.5830 & 0.5853 \\
Reacción Total & 563 & 2.966 & 0.678 & 0.0577 & -0.334 & 0.8868 & 0.8877 \\
\hline
\end{tabular}

el $35 \%$ de la varianza. De esta forma se eliminaron los ítems 3.1 (dimensión estresores), $5.4, \quad 5.5$ y 5.1 (dimensión afrontamiento), originalmente definidos por Barraza y el ítem 5.8 de reciente incorporación (dimensión afrontamiento).

Considerando la solución factorial obtenida, se definen 4 factores a considerar (Tabla 5), Factor 1 que corresponde a Reacciones físicas y psicológicas que incluye a los ítems 4.1, 4.2, 4.3, 4.4, 4.5, 4.6, 4.7, 4.8, 4.9, 4.10, 4.11 y 4.17, Factor 2 que corresponde a estresores e incluye los ítems $3.2,3.3,3.4,3.5,3.6,3.7,3.8$ y 3.9, Factor 4 que corresponde a reacciones del comportamiento social que incluye los ítems 4.10, 4.12, 4.13, $4.14,4.15$ y 4.17 y Factor 3 que corresponde a Estrategias de afrontamiento e incluye los ítems 5.2, 5.3, 5.6, 5.7, 5.9 y 5.10 .

En la Tabla 6 se muestran los estadísticos descriptivos de los factores identificados, así como la confiabilidad de cada uno de ellos. Acerca de la correlación entre los factores, es pertinente mencionar que el factor correspondiente a afrontamiento no mostró correlación con ninguno de los otros factores. El factor de estresores se correlacionó con el factor reacciones físicas y psicológicas y con el factor de reacciones del comportamiento social. Este último, además se 


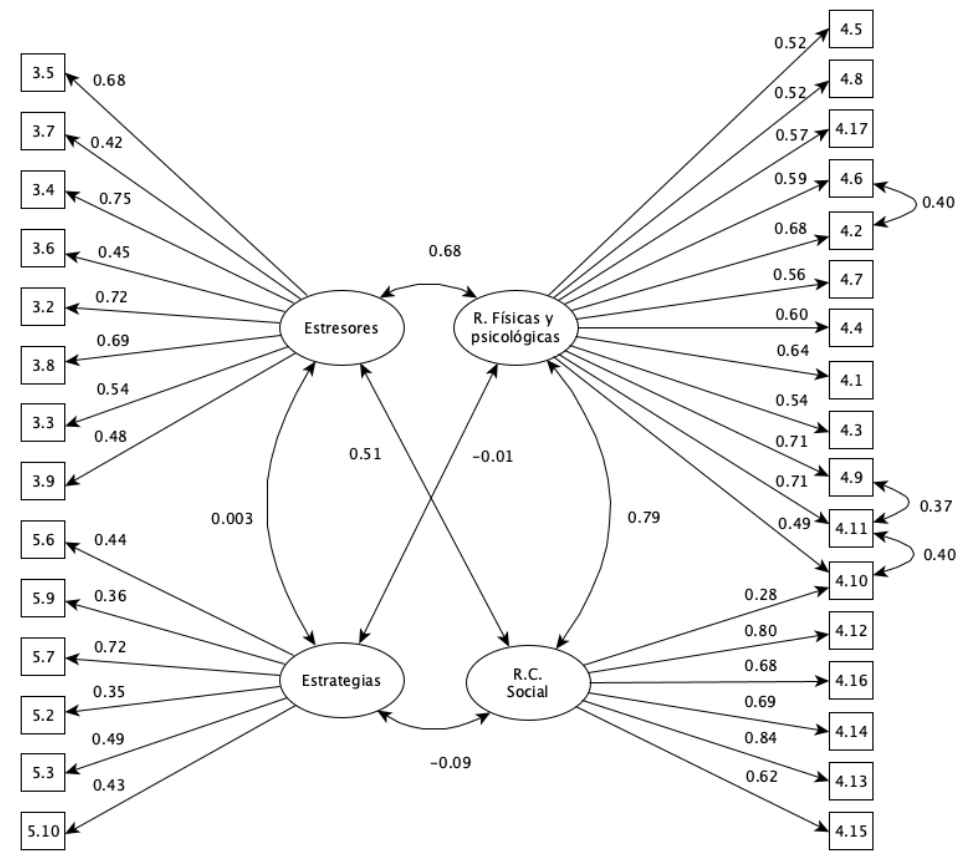

Figura 2. Gráfico de estimadores de los parámetros del modelo en base a 4 factores

correlacionó con el factor de reacciones físicas y psicológicas (Figura 2).

El factor correspondiente a estresores presentó una buena confiabilidad (alfa de Cronbach $=0.78$ y Omega $=0.78$ ) lo cual concuerda con lo reportado previamente (alfa de Cronbach=0.76) (GuzmánCastillo, Sáez, Pérez, \& Castillo-Navarrete, 2018). A su vez, la dimensión de reacción total junto con sus componentes, reacciones físicas y psicológicas y reacciones del comportamiento social, presentaron buenos niveles de confiabilidad con valores de alfa de Cronbach y de Omega de 0.89, 0.85 y 0.84 respectivamente, con una confiabilidad total del instrumento muy buena (alfa de Cronbach=0.90 y Omega=0.90).

En función de lo anterior se reestructuró la dimensión de sintomatología definida por Barraza no sólo en el orden de los ítems, sino que también en su definición. Lo que en su conjunto hemos llamado Reacción Total y que podemos definirla como la reacción del individuo frente a las demandas del entorno que son valoradas como estímulos estresores y que está compuesta por un bloque que contiene las reacciones físicas y psicológicas y por otro bloque con las reacciones del comportamiento social. De esta manera como "Medida de estrés académico", se consideran a los estresores (Factor 2) junto con la reacción total (Factor 1 y Factor 4) además de las estrategias de afrontamiento (Factor 3).
Con relación al análisis factorial confirmatorio, el modelo exploratorio inicial de 37 ítems presentó valores de CFI y TLI menores al 0.95 esperado con un RMSEA significativo (0.066, $p=.000)$. El modelo exploratorio con los 31 ítems finales presentó indicadores de ajuste adecuados, aunque TLI fue menor al 0.95 esperado (0.947 y 0.936 , respectivamente) (Tabla 4).

En la Figura 2 se presentan los estimadores para los parámetros del modelo dónde se puede apreciar las correlaciones entre los factores. El factor 1 (Reacciones físicas y psicológicas) se correlaciona significativamente con el Factor 2 correspondiente a estresores $(\mathrm{r}=0.68)$ y con el factor 4 que corresponde a reacciones del comportamiento social $(\mathrm{r}=0.79)$. A su vez, el factor 2 se correlaciona significativamente con el factor $4(\mathrm{r}=0.51)$, a diferencia del Factor 3 correspondiente a estrategias afrontamiento que no se correlaciona con el factor $1(\mathrm{r}=-0.01)$, factor $2(\mathrm{r}=0.003)$ y factor $4(\mathrm{r}=-0.09)$. Además, se puede apreciar la presencia de altas cargas factoriales a excepción del ítem 4.10 para el factor 4 (reacciones del comportamiento social), lo cual debería ser corroborado con una nueva muestra. Además, éste ítem se correlaciona $(r=0.40)$ con el ítem 4.11 del Factor 1 (Reacciones físicas y psicológicas) y éste ítem a su vez, se correlaciona $(\mathrm{r}=0.37)$ con el ítem 4.9 perteneciente al mismo 
factor, al igual que los ítems 4.2 y 4.6 , que también se correlacionan entre sí $(\mathrm{r}=0.40)$.

\section{Discusión}

No en todas las personas, situaciones que pueden ser consideradas como estresantes generarán estrés, ni tampoco todas las personas reaccionarán de la misma manera, existiendo reacciones que pueden ser consideradas perfectamente normales o bien indicadoras de activación fisiológica, cognitiva o conductual, pudiendo considerarse señales indicadoras de estrés por su persistencia a través del tiempo, siendo difícil asegurar que determinada reacción es producto del estrés y no de otra cosa.

En este contexto, el estrés académico corresponde a una respuesta sistémica de adaptación del organismo ante demandas originadas en un contexto educacional. En cuanto a la prevalencia del estrés académico, los resultados son variados, considerando sólo Latinoamérica, la presencia de estrés académico va desde un $35 \%$ a un $95 \%$ en estudiantes universitarios (Guzmán-Castillo, Sáez, Pérez, \& Castillo-Navarrete, 2018). Una diversidad de factores podría ser atribuidos a estas diferencias, entre ellos la heterogeneidad de la población estudiada en términos del programa de estudios y el año cursado (Bedoya, Perea, \& Ormeño, 2014; Marty, Lavín, Figueroa, Larraín, \& Cruz, 2005; Mosquera et al., 2012; Reducindo et al., 2012). En este contexto, la elección de un determinado instrumento psicométrico resulta una elección metodológica vital, que condicionará la observación indirecta de un fenómeno (Putwain, 2007). En el caso del estrés académico los instrumentos que han sido utilizados van desde cuestionarios confeccionados para un determinado estudio (Bedoya, Perea \& Ormeño, 2014; Marty, Lavín, Figueroa, Larraín, \& Cruz, 2005; Reducindo et al., 2012), inventarios generales de estrés (Marty, Lavín, Figueroa, Larraín, \& Cruz, 2005; Mosquera et al., 2012) hasta instrumentos concebidos para la medición de estrés académico propiamente tal (Barraza-Macías, 2007b; GarcíaRos et al., 2012). Al respecto, en un trabajo anterior abordamos algunas de las propiedades psicométricas del inventario SISCO del estrés académico en estudiantes universitarios del área de la salud, en la Ciudad de Concepción, Chile, así como información relativa a su estructura, evidencia de validez de constructo y confiabilidad, sugiriéndose prescindir de la dimensión de afrontamiento. En consecuencia, en el presente artículo abordamos esta problemática e intentamos adaptar el instrumento.

El presente trabajo implicó tomar ciertas decisiones que impactan al diseño original del instrumento. La decisión de plantear el análisis psicométrico con cuatro factores y no tres, se basó en aspectos tanto teóricos como psicométricos.

En el caso del grupo de ítems correspondientes a estresores, las cargas factoriales, así como los indicadores de confiabilidad fueron muy similares tanto con el análisis psicométrico en base a tres factores como con el análisis a 4 factores (Tabla 3), así como en el ítem eliminado (3.1: la competencia con los compañeros de grupo). Para el grupo de ítems correspondiente a estrategias de afrontamiento, si bien existieron diferencias en cuanto a los ítems eliminados, las cargas factoriales también fueron semejantes (Tabla 3). En relación con los indicadores de confiabilidad estos no fueron buenos, pero sí muy similares (Tabla 6), lo que esta acorde con los que reportamos previamente y permite reafirmar la necesidad de ser cautelosos y estudiar más en detalle la dimensión de afrontamiento (Guzmán-Castillo, Sáez, Pérez, \& Castillo-Navarrete, 2018).

En el caso de los ítems correspondientes a sintomatología definidos en el Inventario SISCO del estrés académico, las diferencias fueron considerables. Si bien en la estructura original planteada por Barraza, la somatización está subdividida en reacciones físicas, reacciones psicológicas y reacciones del comportamiento, el análisis psicométrico en base a tres factores lógicamente los agrupará en un solo factor, mientras que el análisis en base a 4 factores agrupará estos ítems en dos factores diferentes. Lo anterior tiene importancia para el instrumento ya que se agrupan en un factor los ítems sobre los cuales el individuo no tiene mayor control y que corresponde a la reacciones físicas y psicológicas y en el otro factor se agrupan las reacciones que corresponden a la consecuencia conductual de los estresores (y en parte también de las reacciones 
físicas y psicológicas). Desde el punto de vista psicométrico, las cargas factoriales son fuertes, así como también los indicadores de confiabilidad son muy buenos (Tabla 5 y Tabla 6). De esta manera, la reacción del individuo frente a las demandas del entorno que son valoradas como estímulos estresores (reacción total) se corresponde con los dos factores mencionados, los cuales hemos denominado reacciones físicas y psicológicas y reacciones del comportamiento social.

Esta reestructuración de la dimensión de sintomatología definida por Barraza, tanto en la numeración de los ítems como en la definición subyacente a los mismos, involucra centrar la definición de estrés académico no sólo en la evaluación de reacciones físicas, psicológicas y del comportamiento, sino que en el proceso de transacción entre las demandas académicas (y sociales de este proceso) valoradas por el estudiante en función de sus propias metas, valores, experiencias y necesidades. De esta forma, cuando el estudiante percibe (consciente o inconscientemente) un desbalance, sostenido en el tiempo, entre estas demandas y los recursos que están a su disposición para superarlas, comenzará por un lado a expresar en forma fisiológica y psicológica el desbalance producido, modificando su conducta (también consciente o inconscientemente).

La estructura de 4 factores planteada para el inventario SISCO-II del estrés académico presenta un mejor ajuste en comparación con la solución de 3 factores, cuando se considera al análisis factorial exploratorio, los indicadores de confiabilidad y los resultados del análisis factorial confirmatorio.

En consecuencia, considerando por un lado los resultados del análisis psicométrico y por otro, esta perspectiva transaccional planteada, bastante cercana a lo propuesto en su momento por Richard Lazarus (Lazarus, 1990; 2000; Lazarus \& Folkman, 1991), es adecuado plantear con relación al inventario SISCO-II (versión modificada del inventario SISCO del estrés académico):

(i) La dimensión de estresores está conformada por 8 ítems, de los cuales uno es un nuevo ítem (3.8). Es destacable que se eliminó un ítem definido originalmente por Barraza (3.1: la competencia con los compañeros de grupo)
(Barraza-Macías, 2007b). Es curiosa la similitud conceptual del ítem eliminado (3.1) y el nuevo ítem 3.8 (Los compañeros de grupo progresan más rápido en tareas y/o trabajos académicos), si bien es cierto que son ítems conceptualmente diferentes, la preocupación por el progreso más rápido de los compañeros, en cierta medida sí es una forma de competencia con los compañeros de grupo, lo que sugiere la posibilidad de diferencias tanto socioculturales como generacionales de las poblaciones estudiadas.

(ii) Se reestructuró la dimensión de sintomatología definida por Barraza, tanto en el orden de los ítems que la componen, como en su definición quedando compuesta por un bloque que contiene las reacciones físicas y psicológicas y por otro bloque con las reacciones del comportamiento social, es decir lo que en su conjunto hemos llamado Reacción Total. Es pertinente mencionar que el ítem 4.10 (Sentimientos de depresión y tristeza (decaído)) presentó una carga factorial similar (0.44 y 0.41) para reacciones físicas y psicológicas (Factor 1) y para reacciones del comportamiento social (Factor 4), respectivamente. Este ítem, definido originalmente por Barraza, no obstante presentar esta carga cruzada, se considera teóricamente importante y conceptualmente se asignó a reacciones físicas y psicológicas (Factor 1). También es importante mencionar, que el ítem 4.17 (Aumento o reducción del consumo de alimentos) definido originalmente por Barraza e incluido en la dimensión de somatización como una reacción del comportamiento (BarrazaMacías, 2007b), en base a la solución factorial obtenida, es plausible incorporarlo como una reacción física y psicológica (Factor 1).

(iii) Considerando los resultados del análisis psicométrico para la dimensión de afrontamiento (bajo nivel de confiabilidad con un alfa de Cronbach de 0.58 y Omega de 0.59) y en concordancia con lo que ya habíamos vislumbrado previamente (Guzmán-Castillo, Sáez, Pérez, \& Castillo-Navarrete, 2018), se modifica la dimensión de afrontamiento, la cual había quedó conformada por 3 de los 6 ítems propuestos por Barraza (5.2: Elaboración de un plan de ejecución de tareas, 5.3: Elogios a sí mismo y 5.6: ventilación y confidencias (verbalización de la situación que preocupa) y por 3 de los 4 ítems de 
Tabla 7. Inventario SISCO-II del estrés académico

\begin{tabular}{|c|c|}
\hline \multicolumn{2}{|r|}{ Ítem } \\
\hline P1 & Durante el transcurso de este semestre ¿ha tenido momento de preocupación o nerviosismo? \\
\hline $\mathrm{P} 2$ & $\begin{array}{l}\text { Con la idea de obtener mayor precisión y utilizando una escala de } 1 \text { a } 5 \text { señala tu nivel de preocupación o nerviosismo, dónde (1) } \\
\text { es poco y (5) mucho. }\end{array}$ \\
\hline \multicolumn{2}{|r|}{ Estresores } \\
\hline 3.1 & Sobrecarga de tareas y trabajos académicos \\
\hline 3.2 & La personalidad y carácter de los profesores \\
\hline 3.3 & Las evaluaciones de los profesores (exámenes, ensayos, trabajos de investigación, etc.) \\
\hline 3.4 & El tipo de trabajo que te piden los profesores (consulta de mapas, fichas de trabajo, ensayos, mapas conceptuales, etc.) \\
\hline 3.5 & No entender los temas que se abordan en la clase \\
\hline 3.6 & Participación en clase (responder a preguntas, exposiciones, etc.) \\
\hline 3.7 & Tiempo limitado para hacer el trabajo \\
\hline 3.8 & Los compañeros de grupo progresan más rápido en tareas y/o trabajos académicos. $(*)$ \\
\hline \multicolumn{2}{|r|}{ Reacción total: Reacciones Físicas y Psicológicas } \\
\hline 4.1 & Trastornos del sueño (insomnio o pesadillas) \\
\hline 4.2 & Fatiga crónica (cansancio permanente) \\
\hline 4.3 & Dolores de cabeza o migrañas \\
\hline 4.4 & Problemas de digestión, dolor abdominal o diarrea \\
\hline 4.5 & Rascarse, morderse las uñas, frotarse, etc. \\
\hline 4.6 & Somnolencia o mayor necesidad de dormir. \\
\hline 4.7 & Dolores musculares y/o contracturas $(*)$ \\
\hline 4.8 & Reacciones cutáneas (sarpullido, descamación, etc.) (*) \\
\hline 4.9 & Inquietud (incapacidad de relajarse y estar tranquilo) \\
\hline 4.10 & Ansiedad, angustia o desesperación \\
\hline 4.11 & Aumento o reducción del consumo de alimentos \\
\hline \multicolumn{2}{|r|}{ Reacción total: Reacciones del Comportamiento Social } \\
\hline 4.12 & Sentimientos de depresión y tristeza (decaído) \\
\hline 4.13 & Sentimiento de agresividad o aumento de irritabilidad \\
\hline 4.14 & Cambios bruscos de humor $(*)$ \\
\hline 4.15 & Conflictos o tendencia a polemizar o discutir \\
\hline 4.16 & Aislamiento de los demás \\
\hline 4.17 & Desgano para realizar tus labores de estudiante \\
\hline \multicolumn{2}{|r|}{ Estrategias de Afrontamiento } \\
\hline 5.1 & Elaboración de un plan de ejecución de sus tareas \\
\hline 5.2 & Elogios a sí mismo \\
\hline 5.3 & Ventilación y confidencias (verbalización de la situación que preocupa) \\
\hline 5.4 & Intenté sacar algo positivo o beneficioso de la situación estresante $(*)$ \\
\hline 5.5 & Practicar un pasatiempo (act. Física, leer, ver series, redes sociales, etc.) (*) \\
\hline 5.6 & Acompañarse de un ser querido (familia, mascotas, amigos, etc.) (*) \\
\hline
\end{tabular}

reciente incorporación (5.7: intenté sacar algo positivo o beneficioso de la situación estresante, 5.8: practicar un pasatiempo y 5.10: acompañarse de un ser querido).

De esta manera, SISCO-II, la versión modificada del inventario SISCO del estrés académico, queda compuesto por 33 ítems, 08 ítems en la dimensión de estresores, 17 ítems en la dimensión de reacción total (con uno y tres ítems de reciente incorporación, respectivamente) y 06 ítems en la dimensión correspondiente a estrategias de afrontamiento (con 3 ítems de reciente incorporación), más las dos preguntas iniciales. En la Tabla 7 se muestra la estructura de SISCO-II, la cual implica además un cambio en la numeración y en el orden de los ítems.

Si bien, por un lado, la decisión de realizar el análisis psicométrico en base a cuatro factores es determinante para el constructo del inventario SISCO-II del estrés académico, también lo es la decisión de conservar la dimensión de estrategia de afrontamiento, a pesar de sus mediocres características psicométricas.

El afrontamiento puede ser definido como "aquellos esfuerzos cognitivos y conductuales constantemente cambiantes que se desarrollan para manejar las demandas específicas externas o internas que son evaluadas como desbordantes de los recursos del individuo" (Lazarus, 2000; Lazarus \& Folkman, 1991). Al ser un constructo multidimensional, que no es parte constituyente del estrés académico sino un factor asociado que permite moderarlo es dinámico, implicando una permanente evaluación por parte del individuo con el objetivo de implementar estrategias que apunten a acciones orientadas a modificar $\mathrm{o}$ alterar el problema (acción) y que ayuden a regular las respuestas emocionales a las que el problema da lugar (emoción) (Lazarus, 2000; Lazarus \& Folkman, 1991). Es interesante considerar que se eliminaron los ítems 5.4 (práctica religiosa) y 5.5 (búsqueda de información sobre la situación), los 
mismos ítems que en un estudio anterior también fueron eliminados (Guzmán-Castillo, Sáez, Pérez, \& Castillo-Navarrete, 2018). Aunque, Malo y colaboradores refiere una buena confiabilidad para la dimensión de afrontamiento (alfa de Cronbach=0.7) en estudiantes universitarios de Colombia (Malo et al., 2010), esto contrasta con lo encontrado en estudiantes de Chile, probablemente debido a diferencias, tanto geográficas, étnicas como socioculturales, lo cual reafirma que los ítems eliminados tienen que ver más con prácticas socioculturales que pueden diferir entre Colombia y Chile, que con las capacidades propias de cada individuo (GuzmánCastillo, Sáez, Pérez, \& Castillo-Navarrete, 2018). El ítem 5.8 (Consumo de sustancias, café, bebidas energéticas, tabaco, etc.) de reciente incorporación y que también fue eliminado, en su definición teórica es conceptualmente amplio, dejando abierta la opción de respuestas tanto a sustancias lícitas como ilícitas, lo cual obviamente difícilmente sería declarado por los participantes, razón por lo cual no fue sorpresa que se procediera a su eliminación. El otro ítem eliminado de la dimensión de afrontamiento, definido por Barraza, fue el 5.1 (Habilidad asertiva, defender nuestras preferencias 0 sentimientos), lo que indicaría que los estudiantes más que plantearse defender sus preferencias optan por otras estrategias de afrontamiento no presentes en los ítems definidos por Barraza.

Desde el punto de vista psicométrico, la dimensión de estrategias de afrontamiento no presenta buenos indicadores y si bien prescindir de esta dimensión es una alternativa, hacerlo afectaría al constructo teórico definitorio de estrés académico en que se fundamentan tanto el inventario SISCO del estrés académico en su forma original como también el inventario SISCO-II. Además, con el inventario SISCO-II del estrés académico se refuerza la relación entre el constructo teórico y las características psicométricas de las dimensiones de estresores y sintomatología (reacción total), pero se mantiene el cuestionamiento a que los ítems usados sean los adecuados para estudiar estrategias de afrontamiento.

Nuestro cuestionamiento a la dimensión de afrontamiento tiene base no sólo psicométrica, sino que también teórica, al ser un constructo diferente, difícil de medir adecuadamente sólo con 6 ítems, existiendo instrumentos que apuntan específicamente a su medición (Nava et al., 2013). A modo de ejemplo se puede mencionar el inventario Multidimensional de Afrontamiento que mide 5 dimensiones en 70 ítems (Crespo \& Cruzado, 1997), la escala de afrontamiento frente a riesgos extremos que mide 2 factores en 36 ítems (López-Vázquez \& Marván, 2004), el inventario de estrategias de afrontamiento que mide 8 factores en 40 ítems (Cano, Rodríguez, \& García, 2007) y la escala de afrontamiento para adolescentes que mide 18 dimensiones en 80 ítems (Canessa, 2002). Al respecto es indudable que, para una adecuada medición del afrontamiento frente al estrés académico, se requiere de un instrumento diseñado específicamente con ese fin, como, por ejemplo, la escala de afrontamiento del estrés académico (ACEA), instrumento de 23 ítems agrupados en tres factores y con buenas propiedades psicométricas (alfa de Cronbach entre 0.81 y 0.9 ).

Como la dimensión de afrontamiento del instrumento original presenta indicadores psicométricos débiles tanto en lo reportado por Barraza como en nuestro trabajo previo, lo que se mantiene para el inventario SISCO-II del estrés académico, surge la interrogante acerca de las causas de esto. Creemos que la respuesta es posible encontrarla no sólo en diferencia socioculturales, sino que también generacionales. Diferencias socioculturales ya que la forma en que los individuos reaccionan frente al estrés es muy dependiente del contexto, dónde además asoman diferencias generacionales, dadas por la forma en que los individuos actualmente se relacionan con la tecnología y como la utilizan para enfrentar el estrés, utilizando elementos tecnológicos, como plataformas de contenido audiovisual, para películas y/o series de televisión, juegos en línea e interaccione en redes sociales, elementos disponibles sólo durante los últimos años.

El presente estudio tiene varias limitaciones, siendo un aspecto relevante que la muestra está constituida mayoritariamente por individuos del sexo femenino (74.51\%), lo que impide extrapolar a la comunidad estudiantil universitaria en su totalidad. Si bien el tamaño de la muestra fue adecuado tanto para el AFE como para AFC, no lo fue para construir un baremo definitivo de 
SISCO-II. Finalmente, si bien el instrumento está diseñado en la forma de auto reporte, los datos provenientes de éste tienen el riesgo potencial de errores en lo reportado por los individuos o bien sesgos en las muestras.

En conclusión, de la versión modificada del inventario SISCO-EA, planteamos una nueva versión, SISCO-II, que permite la medida de estrés académico y que queda compuesto por un total de 33 ítems distribuidos en 02 preguntas iniciales, 08 ítems en la dimensión de estresores, 17 ítems en la dimensión de reacción total (con uno y tres ítems de reciente incorporación, respectivamente) y 06 ítems en la dimensión de estrategias de afrontamiento (con 3 ítems de reciente incorporación). La estructura de SISCOII implica un cambio en la numeración y en el orden de los ítems, respecto del inventario SISCO del estrés académico.

Los resultados del presente estudio muestran que el inventario SISCO-II tiene un adecuado funcionamiento y es un instrumento válido $\mathrm{y}$ confiable, siendo necesario manejar con cautela la información proveniente de la dimensión de estrategias de afrontamiento. Es por esta razón que consideramos, que por lo menos en Chile, se requieren estudios más acabados sobre las estrategias de afrontamiento utilizadas por los estudiantes universitarios (considerando su complejidad teórica), a fin de complementarla con nuevos ítems acordes con el contexto tecnológico y generacional actual.

Este instrumento derivado de una forma modificada del inventario SISCO del estrés académico surge de la necesidad de adecuar la identificación oportuna de estrés académico en estudiantes universitarios al contexto educativo y tecnológico presentes actualmente en las universidades.

\section{Financiamiento}

Este trabajo fue financiado por los proyectos: OT:2016-6 Facultad de Medicina UDEC, DINREG 01/2018 de la Dirección de Investigación UCSC. Concurso Beca de Doctorado en Chile Conicyt No 21160620.

\section{Referencias}

Barraza, A. (2006). Un modelo conceptual para el estudio del estrés académico. Revista Electrónica de Psicología Iztacala, 9(3), 20.

Barraza, A. (2007a). Estrés académico: Un estado de la cuestión. Revista PsicologiaCientifica.com 9(2).

Barraza, A. (2007b). Propiedades psicométricas del Inventario SISCO del estrés académico. Revista PsicologiaCientifica.com.

Barraza, A., \& Medina, S. (2017). El estrés académico en estudiantes de gastronomía de una universidad privada de la ciudad de Durango, en México. Revista de Psicología Universidad de Antioquia, 8(2), 11-26.

Bedoya, S., Perea, M., \& Ormeño, R. (2014). Evaluación de niveles, situaciones generadoras y manifestaciones de estrés académico en alumnos de tercer y cuarto año de una Facultad de Estomatología. Revista Estomatológica Herediana, 16(1), 15.

Bruna, D., Pérez, M., Bustos, C., \& Núñez, J. (2017). Propiedades psicométricas del inventario de procesos de autorregulación del aprendizaje en estudiantes universitarios chilenos. Revista Iberoamericana de Diagnóstico y Evaluación - e Avaliação Psicológica, 44(2), 77-91.

Buja, A., \& Nermin, E. (1992). Remarks on parallel analysis. Multivariate Behavioral Research, 27(4), 509-40.

Canessa, B. (2002). Adaptación psicométrica de las Escalas de Afrontamiento para Adolescentes de Frydenberg y Lewis en un grupo de escolares de Lima metropolitana. Persona. Recuperado 2 de junio de 2016 (http://www.redalyc.org/articulo.oa?id=14711 8132008).

Cano, F., Rodríguez, L., \& García, J. (2007). Adaptación española del Inventario de Estrategias de Afrontamiento. Actas Españolas de Psiquiatría, 35(1), 29-39.

Correo-Prieto, F. (2015). Estrés académico en estudiantes de medicina de la Universidad Cesar Vallejo, de Piura 2013. Revista del Cuerpo Médico del Hospital Nacional Almanzor Aguinaga Asenjo, 8(2), 80-84.

Cova, F., Bustos, C., Rincón, P., Grandón, P. Saldivia, S., \& Inostroza, C. (2019). 
Propiedades psicométricas de una forma breve del Cuestionario de Parentalidad Alabama en familias de preescolares chilenos. Revista Iberoamericana de Diagnóstico y Evaluación - e Avaliação Psicológica, 51(2).

Crespo, M., \& Cruzado, J. A. (1997). La intervención del afrontamiento: Adaptación española del cuestionario COPE con una muestra de estudiantes universitarios. Análisis y Modificación de Conducta, 23(92), 797-830.

Dyson, R., \& Renk, K. (2006). Freshmen adaptation to university life: Depressive symptoms, stress, and coping. Journal of Clinical Psychology, 62(10), 1231-44.

Espinel, J., Robles, J., \& Álvarez, G. (2015). Factores de riesgo y prevención del estrés académico en estudiantes universitarios de la UNEMI, Milagro. Revista Científica Yachana, 4(2).

Fasce, E., Pérez, C., Ortiz, L., Parra, P. \& Matus, O. (2011). Estructura factorial y confiabilidad de la escala de aprendizaje autodirigido de Fisher, King \& Tague en alumnos de medicina chilenos. Revista Médica de Chile, 139(11), 1428-1434.

García-Ros, R., Pérez-González, F., Pérez-Blasco, J., \& Natividad, L. (2012). Evaluación del estrés académico en estudiantes de nueva incorporación a la universidad. Revista Latinoamericana de Psicología, 44(2), 143154.

Guzmán-Castillo, A., Sáez, K., Pérez, C., \& Castillo-Navarrete, J. L. (2018). Validity and reliability of SISCO Inventory of Academic Stress among health students in Chile". Journal of Pakistan Medical Association, 68(12), 5.

Hair, J., Black, W., Babin, B., \& Anderson, R. (2010). Multivariate data analysis (6th ed.). New Jersey: Prentice Hall.

Horn, J. L. (1965). A rationale and test for the number of factors in factor analysis. Psychometrika, 30(2), 179-85.

Jaimes, R. (2008). Tesis: Validación del inventario SISCO del estrés académico en adultos jóvenes de la Universidad Pontificia Bolivariana, Seccional Bucaramanga. Tesis, Universidad Pontificia Bolivariana, Bucaramanga, Colombia.
Kaiser, H. F. (1974). An index of factorial simplicity. Psychometrika, 39(1), 31-36.

de La Rosa-Rojas, G., Chang-Grozo, S., DelgadoFlores, L., Oliveros-Lijap, L., Murillo-Pérez, D., Ortiz-Lozada, R., Vela-Ulloa, G., \& Carreazo, N. (2015). Niveles de estrés y formas de afrontamiento en estudiantes de Medicina en comparación con estudiantes de otras escuelas. Gaceta Médica México, 151, 443-449.

Lazarus, R. S. (1990). Theory-based stress measurement. Psychological Inquiry, 1(1), 313.

Lazarus, R. S. (2000). Toward better research on stress and coping. American Psychologist, 55(6), 665-73.

Lazarus, R. S., \& Folkman, S. (1991). Estrés y procesos cognitivos. Barcelona: Martínez Roca.

Ledesma, R., Ferrando, P., \& Tosi, J. (2019). Uso del análisis factorial exploratorio en RIDEP. Recomendaciones para autores y revisores". Revista Iberoamericana de Diagnóstico y Evaluación - e Avaliação Psicológica, 52(3).

López-Vázquez, E., \& Marván, M. (2004). Validación de una escala de afrontamiento frente a riesgos extremos. Salud Pública de México, 46(3), 216-221.

Malo, D., Cáceres, G., \& Peña, G. (2010). Validación del inventario SISCO del estrés académico y análisis comparativo en adultos jóvenes de la Universidad Industrial de Santander y la Universidad Pontificia Bolivariana, Seccional Bucaramanga, Colombia". PRAXIS INVESTIGATIVA ReDIE, 2(3), 26-42.

Manrique-Millones, D., Millones-Rivalles, R., \& Manrique-Pino, O. (2019). The SISCO Inventory of Academic Stress: Examination of its psychometric properties in a Peruvian sample". Ansiedad y Estrés, 25(1), 28-34.

Martínez, R., Hernández, M., \& Hernández, M. J. (2006). Psicometría. Madrid: Alianza.

Marty, C., Lavín, M., Figueroa, M., Larraín, D., \& Cruz, C. (2005). Prevalencia de estrés en estudiantes del área de la salud de la Universidad de los Andes y su relación con enfermedades infecciosas. Revista Chilena de Neuro-Psiquiatría, 43(1), 25-32. 
Mejía-Rubalcava, C., Alanís-Tavira, J., ArguetaFigueroa, L., \& Legorreta-Reyna, A. (2012). Academic Stress as a Risk Factor for Dental Caries. International Dental Journal, 62(3), 127-31.

Mosquera, C., Nacad, D., Mezzoni, M., Di Giacomo, Z., Mendible, S., Montplaisier, F., Mata, A., Martínez, A., \& Rodríguez-Morales, A. (2012). Estrés en dos cohortes de estudiantes de medicina de la Escuela 'Luis Razetti', Universidad Central de Venezuela”. Gaceta médica de Caracas, 120(1), 33-39.

Nava-Quiroz, C., Ollua, P., Vega, C., \& Soria, R. (2013). Inventario de Estrategias de Afrontamiento: Una replicación. Psicología y Salud, 20(2), 213-220.

Nunnally, J., \& Bernstein, I. (1994). Psychometric theory. New York: McGraw-Hill.

Oliván-Blázquez, B., Boira-Sarto, S., \& López del Hoyo, Y. (2011). Estrés y otros factores psicológicos asociados en estudiantes de fisioterapia. Fisioterapia, 33(1), 19-24.

Pérez, C., Ortiz, L., Fasce, E., Parra, P., Matus, O., Mccoll, P., Torres, G., Meye,r A., Marquez, C., \& Ortega, J. (2015). Propiedades psicométricas de un cuestionario para evaluar expectativas académicas en estudiantes de primer año de Medicina. Revista Médica de Chile, 143, 1459-1467.

Putwain, D. (2007). Researching academic stress and anxiety in students: some methodological considerations. British Educational Research Journal, 33(2), 207-19.

Reducindo, M., Cortés, P., Cabañas, M., Godínez, F., González, M., \& Landero, R. (2012). Factores estresantes asociados al hiperestrés en estudiantes universitarios de la ciudad de Chilpancingo, Guerrero, México. Summa Psicológica UST (En línea), 9(1), 15-21.

Selye, H. (1973). The evolution of the stress concept: The originator of the concept traces its development from the discovery in 1936 of the alarm reaction to modern therapeutic applications of syntoxic and catatoxic hormones. American Scientist, 61(6), 692-99.

Tabachnick, B., \& Fidell, LS. (2006). Using multivariate statistics. New York: Harper and Row.
Tafet, G. (2008). Psiconeurobiología de la resiliencia. Acta Psiquiátrica y Psicológica de América Latina, 54(2), 000-000.

Tafet, E., \& Nemeroff, C. (2016). The links between stress and depression: Psychoneuroendocrinological, genetic, and environmental interactions. The Journal of Neuropsychiatry and Clinical Neurosciences, 28(2), 77-88. 